\title{
Aucubin promoted neuron functional recovery by suppressing inflammation and neuronal apoptosis in a spinal cord injury model
}

\section{Shining Xiao}

First Affiliated Hospital of Nanchang University

Nanshan Zhong

First Affiliated Hospital of Nanchang University

Quanming Yang

Ningbo City First Hospital

Anan Li

First Affiliated Hospital of Nanchang University

Weilai Tong

First Affiliated Hospital of Nanchang University

\section{Yu Zhang}

First Affiliated Hospital of Nanchang University

\section{Geliang Yao}

First Affiliated Hospital of Nanchang University

\section{Shijiang Wang}

First Affiliated Hospital of Nanchang University

Jiaming Liu

First Affiliated Hospital of Nanchang University

Zhili Liu ( $D$ liuzhiliyfy@163.com )

The First Affiliated Hospital of Nanchang University https://orcid.org/0000-0001-5199-2589

\section{Research article}

Keywords: Spinal cord injury, Neuronal apoptosis, Neuroinflammation, Aucubin, TLR4/NF-kB pathway

Posted Date: February 23rd, 2022

DOI: https://doi.org/10.21203/rs.3.rs-1342741/v1

License: (1) (1) This work is licensed under a Creative Commons Attribution 4.0 International License.

Read Full License 


\section{Abstract}

Spinal cord injury $(\mathrm{SCl})$ can cause severe motor impairment. Post-SCl treatment has focused primarily on secondary injury, with neuroinflammation and neuronal apoptosis as the primary therapeutic targets. Aucubin ( $\mathrm{Au}$ ), a Chinese herbal medicine, exerts anti-inflammatory and neuroprotective effects. The therapeutic effects of $\mathrm{Au}$ in $\mathrm{SCl}$ have not been reported. We showed that Au can promote functional recovery after $\mathrm{SCl}$. Recovery may occur through the toll-like receptor 4 (TLR4)/nuclear factor kappa B (NF$\mathrm{KB}$ ) pathway to promote $\mathrm{M} 2 / \mathrm{M} 1$ polarization in microglia and inhibit mitochondrial dysfunction to reduce neuronal apoptosis. These biochemical changes result in reduced secondary injury and facilitate axon regeneration. Therefore, Au may be a promising post-SCI therapeutic medication.

\section{Introduction}

Spinal cord injury $(\mathrm{SCl})$ is a terrible neurological condition that usually results in lifelong paraplegia and is associated with substantial physical and financial suffering (K. T. Huang \& Lu, 2021; Q.-M. Pang et al., 2021; Rivers et al., 2018). However, there are no effective treatments for SCl. The pathological process of $\mathrm{SCl}$ can be divided into primary and secondary injuries (C. Wang et al., 2021; Xiao et al., 2021). Primary $\mathrm{SCl}$, which includes in situ structural damage, vascular rupture in the lesion area, and local bleeding around the lesion area, is caused by traumatic events (e.g., vehicle accidents or falling from a building) (Sámano \& Nistri, 2019; Saunders, Clarke, Tate, Forchheimer, \& Krause, 2015; Tran, Warren, \& Silver, 2018). Secondary injuries are a group of disorders that develop following the primary injury, including severe inflammation, apoptosis, oxidative stress, ischemia, hypoxia, and axon damage (Tran et al., 2018). Repair of spinal cord tissue is hampered by secondary damage. Studies have shown that effective treatment of secondary damage is critical to SCl recovery (Shen et al., 2021; C. Wang et al., 2019), and inflammation and apoptosis are essential regulatory targets for prevention of secondary injury (Lin et al., 2020).

Inflammation is a critical effector of the healing process, but chronic inflammation exacerbates secondary complications following SCI (Q. Pang et al., 2021). Microglia are central nervous system macrophages that can express pro-inflammatory (M1) and anti-inflammatory (M2) phenotypes (Ronaldson \& Davis, 2020). When suffering from SCl, microglia can be activated quickly. Proinflammatory mediators (e.g., cyclooxygenase (COX)-2, inducible nitric oxide synthase (iNOS), tumor necrosis factor (TNF)- $a$, Interleukin (IL)-1, and interleukin (IL)- 6 etc.) are released by activated M1 phenotype microglia, resulting in an inflammatory response (Jin et al., 2019). At the same time, SCI results in disruption of the blood-spinal-cord barrier. This disruption results in infiltration of peripheral immune cells (e.g., macrophages) at the injury site, which aggravates the inflammatory response (Park et al., 2019). After SCl, secondary injury increases and hampers the rehabilitation of linked functions due to inflammation inside and outside the central nervous system (Gong et al., 2020). Studies have shown that increasing the M2/M1 polarization ratio in microglia/macrophages after SCl (Gaojian et al., 2020), or transplanting M2 phenotype microglia/macrophages to the injured spinal cord (Luo et al., 2021; Ma et al., 2015), can promote functional recovery following SCI. 
Neuronal apoptosis results in neurological dysfunction and is associated with poor prognosis (Moujalled, Strasser, \& Liddell, 2021; Ramer, Ramer, \& Bradbury, 2014). Reducing neuronal apoptosis can promote neurorehabilitation, neuroplasticity, and axonal regeneration, and has become a strategy for treatment of SCI (Williams et al., 2014). Recent studies have shown that reducing the level of neuronal apoptosis can improve the recovery of motor function in rats that experienced SCI (Giraldo et al., 2021; Yuan et al., 2021). Therefore, promoting $M 1$ to $M 2$ polarization of microglia/macrophages and inhibiting neuronal apoptosis may promote functional recovery following SCl.

Studies have shown that anti-neuroinflammatory medications and anti-apoptotic therapies have been shown to improve behavioral functions in a variety of animal models of SCl (Lin et al., 2020; Xiao et al., 2021). However, few medications can enhance the $M 2 / M 1$ polarization ratio in microglia/macrophages and prevent neuronal death. Therefore, anti-inflammatory and neuroprotective natural Chinese herbal medications have been extensively studied. Aucubin ( $\mathrm{Au}$ ) belongs to the iridoid glycoside family and is found in a variety of plant species (H. Wang et al., 2020). A number of studies have shown that Au exerts potent anti-inflammatory (Gao et al., 2021), anti-oxidant (Yang et al., 2018), and anti-tumor effects (Kim, Sim, Shin, \& Kim Kwon, 2014), and protects liver (S. Zhang et al., 2020) and brain tissue (H. Wang et al., 2020). However, treatment of rats with Au following SCl has not been studied. In this study, we showed that Au promoted functional recovery of rats who subjected to SCl. Besides, we evaluated the mechanisms by which Au regulated microglia/macrophage polarization in $\mathrm{SCl}$ and exerted neuroprotective effects.

\section{Methods}

\subsection{SCI model establishment and therapy}

We purchased 36 adult female SD rats weighing approximately $220 \mathrm{~g}$ from Tianqin Biotechnology (Changsha, China). Then the rats were randomly divided into three groups: sham operation (Sham group), $\mathrm{SCl}$ caused by extrusion ( $\mathrm{SCl}$ group), $\mathrm{SCl}$ with $\mathrm{Au}(40 \mathrm{mg} / \mathrm{kg}$ ) treatment (Au group). We anesthetized the rat with $1 \%(\mathrm{w} / \mathrm{v})$ sodium pentobarbital $(40 \mathrm{mg} / \mathrm{kg})$ before removing the lamina at the $T 9$ level to expose the spinal cord and modeling the spinal cord injury. After that, we used a vascular clip ( $15 \mathrm{~g}$ force, Oscar) to compress the spinal cord tissue for 1 minute to create a SCl model. The rats in the Au group were injected intraperitoneally with $\mathrm{Au}(40 \mathrm{mg} / \mathrm{kg})$ every day following the $\mathrm{SCl}$ operation, whereas the other groups received the same amount of normal saline. Furthermore, bladder squeezing was performed on the rats in the spinal cord injury groups twice daily to allow for urination.

\subsection{Behavioral evaluation}

Motor function was assessed using the Basso, Beattie, and Bresnahan (BBB) scale, and footprint analysis. For the BBB scale, on days $0,1,3,7,14$, and 28 after surgery we placed the rats in an open environment and three independent observers who were unaware of the experimental procedure observed hind limb movement. The test took approximately 3 minutes, and the results were evaluated on a scale of 0 to 21 points (totally paralyzed hindlimb to normal mobility) (C. Wang et al., 2021). For footprint 
analysis, we dyed the forelimbs and hind limbs of rats with black ink and red ink, respectively, and then had them walk on white paper, and evaluated the imprints (Fan et al., 2018).

\subsection{Cell viability evaluation}

The CCK8 kit (Beyotime, China) was used to measure the toxicity of Au (HPLC purity $>98 \%$, Yaji Biotechnology, China) in BV2 cells. BV2 cells were plated at a density of 5000 cells per well in a $96-$ well plate. After a 24-hour incubation period with $\mathrm{Au}$ at concentrations of $0,0.1,1,10,100,500$, or $1000 \mu \mathrm{M}$, the BV2 cells were incubated for 2 hours with the CCK-8 kit before being measured the optical density (OD) at $450 \mathrm{~nm}$.

We then evaluated the effects of Au on Tert-butyl hydroperoxide (TBHP; Sigma)-induced neuronal apoptosis. PC12 cells were pretreated for 12 hours with $\mathrm{Au}(0,25,50$, or $100 \mu \mathrm{M})$ and subsequently treated for 4 hours with TBHP $(0$ or $100 \mu \mathrm{M})$. The cells were analyzed using the CCK8 kit and OD was measured at $450 \mathrm{~nm}$.

\subsection{Cell culture}

The BV2 microglia and PC12 cells were purchased from Cell Biology (Shanghai, China). The cells were resuspended in high-glucose DMEM medium (BV2 cells) and 1640 medium (PC12 cells), each containing $10 \%$ FBS (Gibco, Thermo). The cells were then cultured at $37^{\circ} \mathrm{C}$ in a humidified incubator containing $5 \%$ $\mathrm{CO}_{2}$. Every two days, the new medium was replenished.

\subsection{Live-dead cell staining}

A Calcein-AM/PI (Solarbio, China) double-staining experiment was utilized to assess the effect of Au on TBHP-induced PC12 cells. PC12 cells were planted in 6-well plates. After that, Au (0 or $100 \mu \mathrm{M})$ was pretreated for 12 hours before being incubated for 4 hours with TBHP $(100 \mu \mathrm{M})$. Following that, PC12 cells were gently washed three times with $1 \times$ Assay Buffer to eliminate any leftover active esterase, and then incubated with Calcein-AM and PI combination for 15 minutes at $37^{\circ} \mathrm{C}$. The photos are then captured using a fluorescence microscope (ZEISS).

\subsection{Immunofluorescence staining}

A round glass slide with an 18-mm diameter was placed on a 12-well plate, and BV2 cells were seeded on the round glass slide, in vitro study. The cells were treated with Au ( 0 or $100 \mu \mathrm{M})$ for 12 hours, then incubated with or without Lipopolysaccharide (LPS; $1 \mu \mathrm{g} / \mathrm{mL}$; Sigma) for 24 hours. The cells were then fixed with $4 \%$ paraformaldehyde (PFA) for 15 minutes then permeabilized with $0.5 \%$ Triton $X-100$ for 30 minutes. The slide was blocked for 1 hour at $37^{\circ} \mathrm{C}$ with $5 \%$ bovine serum albumin (BSA), then cultured overnight at $4^{\circ} \mathrm{C}$ with the following primary antibodies: anti-Arg-1 (CST), anti-iNOS (CST), anti-NF-kB p65 (CST), anti-MAP2 (CST), and anti-Bcl-2 (CST). On the next day, the nuclei were stained with DAPI for 5 minutes before incubation with the appropriate secondary antibodies for 1 hour at $37^{\circ} \mathrm{C}$. The slides were then sealed with nail polish. A fluorescent microscope (ZEISS) was employed to visualize the cells. 
In order to collect spinal cord tissue slices for immunofluorescence staining, we chose some rats on the 3rd and 28th days after $\mathrm{SCl}$. The rats were anesthetized with chloral hydrate, and the left ventricle was perfused with $0.9 \% \mathrm{NaCl}$ before being fixed with $4 \%$ PFA. The lesion's spinal cord tissue is then removed, dehydrated, and embedded. Following that, a longitudinal paraffin slices with $5 \mu \mathrm{m}$ thickness were prepared. Following antigen retrieval, paraffin slices were blocked for 1 hour at $37^{\circ} \mathrm{C}$ with $5 \% \mathrm{BSA}$ before being treated overnight at $4^{\circ} \mathrm{C}$ with various primary antibodies as follows: anti-GFAP (Abcam), anti-CD68 (Abcam), anti-Arg-1(CST), anti-NeuN (Abcam), anti-Cleaved Caspase 3 (CST), anti-MAP2 (CST) and antiGAP43(CST). The following methods are identical to those used in vitro.

\subsection{TUNEL staining}

Cell apoptosis can be detected using TUNEL (Keygen Biotech, China) staining. We permeabilized paraffin sections with $0.5 \%$ Triton $\mathrm{X}-100$ for 10 minutes at room temperature following antigen retrieval. The paraffin sections were then incubated with a mixture of labeling solutions (1X Labeling Solution and $\mathrm{TdT}$ ), then with DAPI for nuclear staining. The cells were visualized using a fluorescence microscope (ZEISS).

\subsection{Western blot}

Total protein is isolated from cells and spinal cord tissue. The extract was quantified with BCA reagent and equilibrated to the same volume. The same amount of protein was loaded into the combs of different SDS-polyacrylamide gels before electrophoresis. Then transfer the protein bands to a polyvinylidene fluoride (PVDF) membrane, and block with $5 \%$ skimmed milk powder for 2 hours and then incubate overnight at $4^{\circ} \mathrm{C}$ with appropriate primary antibodies as follows: anti-TLR4 (CST), anti-MyD88 (Proteintech), anti-IKBa (CST), anti-p-IKBa (CST), anti-p65 (CST), anti-p-p65 (CST), anti-GAP43 (CST), antiMAP2 (CST), anti-Arg-1(CST), anti-iNOS (Proteintech), anti-COX-2 (Abcam), anti-NeuN (Abcam), antiCleaved Caspase 3 (CST), anti-Bcl-2 (Proteintech), anti-BAX (Proteintech), anti- $\beta$-Actin (Proteintech) and anti-GAPDH (Abcam). After the next day, the bands were treated with the matching secondary antibodies for 2 hours. Subsequently, the band was detected by the imaging system (Tanon), and the ImageJ software was used for quantitative analysis.

\subsection{Histological staining}

Paraffin slices ( $5 \mu \mathrm{m}$ thickness) were dewaxed, then stained with hematoxylin and eosin (H\&E) as described previously (Zhao et al., 2013). All images were captured using an optical microscope (ZEISS).

\subsection{Statistical analysis}

The mean \pm standard deviation was used to express all results. The data from each group were analyzed using GraphPad Prism 7.0. Statistical significance was determined by one-way analysis of variance (ANOVA) followed by Tukey's multiple comparisons. $P<0.05$ indicates statistical significance.

\section{Results}




\subsection{Au promoted recovery of motor function in rats following SCl}

Behavior (footprint test and BBB locomotion score) and pathology were used to assess functional recovery following $\mathrm{SCl}$. Hind limb trajectories were strikingly different among the groups in the footprint test. After 28 days, compared to the clear footprints of the Sham rats, the hind limbs of the $\mathrm{SCl}$ rats showed two obvious drag marks (black ink), while the $\mathrm{SCl}$ rats that received Au therapy showed some coordinated movement, as evidenced by several black footprints (red arrows; Fig. 1D). Moreover, the BBB score showed that the rats that were subjected to SCl lost motor function on the first day after surgery, and function of the hind limbs returned gradually. Interestingly, the Au therapy group's movement of the hind limbs was clearly superior to the SCI group's (Fig. 1E). Staining with H\&E at 28 days showed that the cystic cavity was much smaller in the Au treatment group than that in the SCl group (Fig. 1F and G). These results indicated that Au may improve pathology and motor function in rats that are subjected to $\mathrm{SCl}$.

\subsection{Au inhibited the inflammatory response after SCl by promoting M2/M1 polarization in microglia/macrophages}

To explore the effects of Au on microglia/macrophage polarization after SCl, we selected rats on the third day after surgery for evaluation. Immunofluorescence staining showed that CD68 microglia/macrophages (M1 phenotype) in the $\mathrm{SCl}$ group were concentrated in the injured area and in the surrounding normal (green), and Au treatment visibly reduced the number of activated

microglia/macrophages in the lesion area and in the surrounding tissue (Fig. 2A and C). Additionally, the protein levels of CD68, iNOS, and COX-2 after Au treatment were significantly lower than those in the SCl group (Fig. 2D-H), which indicated that Au inhibited polarization to the M1 phenotype and reduced the inflammatory response following SCl. To further evaluate M2 polarization after SCl, we used Arg-1 (a M2 microglia/macrophage phenotype marker) immunofluorescence staining to evaluate the distribution of positive cells in the injured area. The results showed that compared with the SCI group, the Sham group hardly saw M2 phenotype microglia/macrophage, while the Au treatment group had more related M2positive cells (Fig. 2B and C). Furthermore, high Arg-1 protein expression in the Au group also showed that Au treatment promoted M2 polarization (Fig. 2D and E). These results may have been due to the presence of a small population of M2 polarized microglia, even following SCI (Lin et al., 2020; Zeng et al., 2019). Therefore, the number of M2 microglia/macrophages in the SCl group was higher than that in the Sham group. Furthermore, the infiltration of immune cells into the injured area increased, and Au treatment significantly promoted M2 polarization, which resulted in a greater number of M2 polarized microglia/macrophages in the Au treatment group. These results indicated that Au promoted M2/M1 polarization after $\mathrm{SCl}$, which resulted in decreased inflammation.

\subsection{Au increased the M2/M1 polarization ratio in microglia}


We first evaluated Au toxicity in BV2 cells. As shown in Fig. 3A, treatment of BV2 cells with different concentrations of $\mathrm{Au}(0,0.1,1,10,100,500$, and $1000 \mu \mathrm{M})$ for 24 hours resulted in no apparent toxicity below $100 \mu \mathrm{M}$. Viability of BV2 cells was significantly lower following treatment with $500 \mu \mathrm{M}$ or $1000 \mu \mathrm{M}$ Au. Therefore, we treated cells with $100 \mu \mathrm{M}$ or less.

Next, we studied the effect of LPS $(1 \mu \mathrm{g} / \mathrm{mL})$ induction on release of pro-inflammatory mediators in vitro. An amoebic alteration (Fig. 3B) in cell shape (red arrow indicated) was observed after LPS stimulated BV2 cells for 24 hours, which showed that BV2 cells were activated to the M1 phenotype. Western blot results showed that the expression levels of pro-inflammatory mediators (e.g., iNOS and COX-2) in BV2 cells after LPS treatment increased in a time-dependent manner within 24 hours (Fig. 3C-E). Furthermore, immunofluorescence staining of iNOS (Fig. 3F and G) agreed with the western blot results. As iNOS and COX-2 are M1 microglial markers, these results indicated that stimulation of BV2 cells with LPS promoted M1 polarization with a peak at 24 hours.

To investigate the influence of Au on microglia polarization, BV2 cells were pretreated with Au for 12 hours, then stimulated with LPS $(1 \mu \mathrm{g} / \mathrm{mL})$ for 24 hours. Figure 4A-C shows that pretreatment with Au (25, 50 , and $100 \mu \mathrm{M})$ reduced the LPS-induced increases in iNOS and COX-2 levels, which agreed with the results of iNOS immunofluorescence analysis (Fig. 4D and E). The results indicated that Au could prevent M1 polarization of microglia. Western blot and immunofluorescence analysis showed that Au pretreatment increased the expression of M2 microglia-related markers (Arg-1) (Fig. 4G-I). In summary, these results indicated that Au inhibited M1 polarization of microglia and promoted the level of polarization of M2 phenotype microglia in a dose-dependent manner.

\subsection{Au inhibited release of LPS-induced pro-inflammatory mediators by regulating the TLR4/NF-KB pathway}

The toll-like receptor 4 (TLR4)-myeloid differentiation protein-88 (MyD88) signaling pathway has been closely related to inflammation in numerous studies (Bayan, Yazdanpanah, \& Rezaei, 2021; Koushki et al., 2021). To determine whether Au suppressed LPS-induced microglial activation via the TLR4-mediated MyD88 pathway, we pretreated BV2 microglia with Au $(0,25,50$, and $100 \mu \mathrm{M})$ for 12 hours before stimulating them with LPS $(1 \mu \mathrm{g} / \mathrm{mL})$ for 24 hours. Treatment with LPS resulted in increased protein expression levels of TLR4 and MyD88 in BV2 microglia, as determined using western blot. Treatment with Au reversed LPS-induced expression of TLR4 and MyD88 in a concentration-dependent manner (Fig. 5AC). These findings were consistent with the anti-inflammatory effects of Au (Fig. 4A-C). These results indicated that Au reduced inflammation through inhibition of the TLR4-MyD88 pathway.

Furthermore, studies have shown that the pro-inflammatory response mediated by the TLR4-MyD88 signaling pathway is linked to a number of other signaling pathways, including nuclear factor kappa $B$ (NF-kB) pathway (R. Li et al., 2021; L. Wang, Zhang, Li, Xu, \& Chen, 2021). We evaluated whether Au inhibited the release of pro-inflammatory mediators in microglia via the NF-KB pathway. Western blot showed that LPS greatly increased the phosphorylation levels of inhibitor kappa Ba (IKBa) and p65 in microglia, and Au dramatically suppressed this effect (Fig. 5D-F). We used PDTC, an NF-kB pathway 
inhibitor, to further investigate the role of NF-KB signaling in Au-mediated reductions in release of proinflammatory mediators. BV2 microglia were pretreated for 2 hours with or without PDTC $(10 \mu \mathrm{M})$, treated for 12 hours with $\mathrm{Au}(25,50,100 \mu \mathrm{M})$, then cultured in LPS-containing medium for 24 hours. As shown in Fig. 5G-I, treatment with PDTC and Au inhibited phosphorylation of IKBa and p65 more than that observed with PDTC or Au alone. We also used Western blot to observe if Au affects NF-KB signaling to alter the LPS-induced pro-inflammatory response. When compared to the effects of PDTC or Au alone, coincubation with PDTC and Au visibly reduced iNOS and COX-2 protein expression levels (Fig. $5 \mathrm{~J}$ and K). These findings were further validated by immunofluorescence staining, which showed that the combination of PDTC and Au effectively prevented LPS-induced nuclear translocation of NF-KB p65 to the nucleus (Fig. $5 \mathrm{~L}$ ). These results demonstrated that the anti-inflammatory effects of Au occurred through modulation of the NF-kB pathway.

\subsection{Au reduced neuronal apoptosis in vivo and in vitro}

Studies have shown that reducing neuronal apoptosis promoted functional recovery after $\mathrm{SCl}$ (W. Huang et al., 2021; Mortezaee, Khanlarkhani, Beyer, \& Zendedel, 2018; Y. Wu et al., 2021). We found that administration of Au reduced cell apoptosis in $\mathrm{SCl}$, as determined using TUNEL staining (Fig. 6A and B). Furthermore, western blot analysis showed that Au treatment partially restored NeuN (neuronal marker) protein expression (Fig. 6C and D). These results indicated that Au treatment inhibited SCl-induced neuronal apoptosis. We further evaluated the effect of Au on neuronal apoptosis through evaluation of expression of apoptotic proteins. Compared with the Sham group, the $\mathrm{SCl}$ group had distinctly increased expression of mitochondrial pro-apoptotic proteins (Bax and cleaved caspase-3), and decreased expression of mitochondrial anti-apoptotic proteins (Bcl-2). Treatment with Au partially reversed these changes in protein expression (Fig. 6E and F). Staining for cleaved caspase-3 produced results that agreed with those obtained by western blot (Fig. $6 \mathrm{G}$ and $\mathrm{H}$ ). These results indicated that administration of $\mathrm{Au}$ could reduce neuronal apoptosis by improving mitochondrial dysfunction after SCl.

To further evaluate the effects of Au on apoptosis, PC12 cells were treated with TBHP to induce oxidative stress and to trigger neuronal apoptosis. Cell viability was determined using the CCK8 kit, and the results showed that Au treatment significantly reversed TBHP-induced neuronal apoptosis (Fig. 7B). We then measured the levels of the mitochondrial anti-apoptosis-related protein $\mathrm{Bcl}-2$. The results showed that $\mathrm{Bcl}-2$ expression was evidently lower in the TBHP group than that in the CON group. However, Bcl-2 level was partially recovered in the Au treatment group (Fig. 7C and D). Furthermore, of the results of staining for Bcl-2 agreed with those obtained using western blot (Fig. 7E and F). Double-staining with calcein-AM and PI also showed that Au inhibited TBHP-induced apoptosis in PC12 cells (Fig. 7G and H). Microscopic analysis showed that PC12 cells in the TBHP group underwent early apoptosis (permeability was clearly increased, and the cell bodies and axons had contracted), and cell shape was partially restored following Au treatment (Fig. 7l). These results indicated that Au could reverse neuronal apoptosis and enhance neuronal survival by improving mitochondrial dysfunction in vivo and in vitro.

\subsection{Au promoted axon regeneration after $\mathrm{SCl}$ in rats}


We measured the expression of an axon microtubule component, MAP2, in spinal cord tissue using immunofluorescence staining. The rostral and caudal axons in the $\mathrm{SCl}$ group showed sparse distribution and low-intensity staining. In contrast, Au treatment resulted in a more regular distribution of MAP2positive axons, with increased density and staining intensity, and evidence of axonal growth toward the injured area (Fig. 8B and C). These results showed that Au promoted axon regeneration and extension following SCl. Additionally, we evaluated extension of nerve filaments in the injured spinal cord by GAP43 staining. Compared with the $\mathrm{SCl}$ group, the Au group had significantly higher expression of GAP43 nerve fibers at the rostral and caudal regions, and these nerve fibers appeared as more punctate or tubular (Fig. 8D and E). Furthermore, the expression levels of MAP2 and GAP43 showed that Au treatment reversed axonal damage induced by $\mathrm{SCl}(\mathrm{Fig}$. 7F-H). These results demonstrated that Au can promote axon regeneration in damaged spinal cord in vivo.

\section{Discussion}

$\mathrm{SCl}$ is a severely debilitating condition that results in significant loss of sensory and motor function (Álvarez et al., 2021; Hellenbrand et al., 2021; Walsh, Wychowaniec, Brougham, \& Dooley, 2021). Extensive research has focused on identification of medications that can promote functional and pathological recovery following spinal cord injury (Jiang et al., 2021; C. Wang et al., 2018; Zheng et al., 2020). We studied a Chinese herbal medicine called $\mathrm{Au}$, which has a strong therapeutic effect on $\mathrm{SCl}$, and explained the mechanism by which Au promoted recovery of motor function in rats with $\mathrm{SCl}$. The average hindlimb score of the SCl group was about 6 points, while the average hindlimb score in the Au group was about 12 points (Fig. 1E). These results were consistent with the general trend of the footprint analysis results (Fig. 1D). This finding demonstrated that Au promoted rehabilitation of hindlimb motor function following SCl. Furthermore, H\&E staining showed that Au reduced the area of cystic cavities compared to that in the $\mathrm{SCl}$ group (Fig. 1F and G), showing that Au aided functional recovery following $\mathrm{SCl}$.

Secondary injury, which typically manifests as severe inflammation and neuronal apoptosis (Lin et al., 2020; J. Zhang et al., 2021), is the principal pathogenic process that aggravates tissue damage and hampers recovery of motor function following SCI (C. Wang et al., 2021; C. Wu et al., 2021). Because secondary injury is a critical therapy window, several studies have focused on reducing inflammation and neuronal death following SCI to aid functional recovery (Macks, Jeong, \& Lee, 2021; Pelisch, Rosas Almanza, Stehlik, Aperi, \& Kroner, 2020). Microglia become over-activated if the central nervous system is injured, resulting in the release of a large number of inflammatory mediators (Boillée, 2021; Scott, Bedi, Olson, Sears, \& Cox, 2021). Generation of these mediators is uncontrolled, and is accompanied by the release of neurotoxic substances such as free radicals and acute-phase proteins, and infiltration of inflammatory cells (Chen, Zhong, \& Li, 2019; C. Wang et al., 2018). Inflammatory mediators and chemokines worsen secondary damage, resulting in a negative impact on recovery following SCI (Pan, Lin, Liu, \& Chen, 2022; Q. Pang et al., 2021). A large number of studies have shown that reducing central nervous system inflammation and peripheral inflammatory cell infiltration (e.g., macrophage) can significantly reduce secondary damage and promote functional recovery following SCI (Gong et al., 2020; C. Wang et al., 2018). After SCl, microglia/macrophages not only polarize into M1 cells causing 
inflammation, but they may also partially polarize into $\mathrm{M} 2$ cells, which have anti-inflammatory properties (Liu et al., 2021; Xiao et al., 2021). The polarized orientation of microglia/macrophages after SCl is strongly skewed toward the M1 phenotype (Gong et al., 2020). Studies have shown that modulating the polarization of microglia/macrophages between M1 and M2 following SCl can significantly impact the inflammatory response (Lin et al., 2020; Xiao et al., 2021). Therefore, promoting M2 polarization of microglia/macrophages in the injured spinal cord may significantly improve SCl recovery. After SCl, the number of M1 phenotype microglia/macrophages increased at the injury site and permeated the surrounding area. Surprisingly, the number of M1 cells in the Au group was significantly reduced and mostly restricted to the lesion area (Fig. $2 \mathrm{~A}$ and $\mathrm{C}$ ), while the protein levels of the relevant $\mathrm{M} 1 \mathrm{cell}$ markers (CD68, iNOS, and COX-2) were also clearly reduced (Fig. 2D-H). More notably, the Au group had much greater positive numbers and protein levels of $\mathrm{M} 2 \mathrm{microglia} /$ macrophage than the SCl group (Fig. 2B-E). In addition, we used LPS to activate BV2 cells in vitro to mimic neuroinflammation. The results showed that LPS treatment polarized BV2 cells to the M1 phenotype and enhanced release of pro-inflammatory mediators, with a peak at 24 hours (Fig. 3). Pretreatment with Au reduced LPS-induced M1 polarization of $\mathrm{BV} 2$ cells and promoted M2 polarization (Fig. 4). These results were consistent with the effects of $\mathrm{Au}$ treatment on microglia/macrophages in vivo. We showed that Au treatment increased the M2/M1 ratio for microglia/macrophages and reduced inflammation in vivo and in vitro.

Toll-like receptor 4, a member of the toll-like receptor family, plays an important role in cellular immunity (Li, Jiang, \& Wang, 2021). Studies have shown that TLR4 is abundantly expressed in microglia and is associated with pathogenesis of neuroinflammation (Wan et al., 2017). Toll-like receptor 4 can bind to various signaling proteins, such as MyD88, to activate downstream signals, resulting in immune system activation and increased expression of inflammatory genes (Jeong et al., 2014). We showed that LPS increased TLR4 and MyD88 expression in microglia. In contrast, Au clearly reversed the LPS-induced increase in TLR4 and MyD88 levels (Fig. 5A-C). These data were consistent with results showing that Au inhibited release of pro-inflammatory mediators from microglia in vitro (Fig. 4A-C). These results suggest that Au blocked the TLR4-MyD88 signaling pathway, thereby inhibiting the neuroinflammation in LPSactivated microglia. The TLR4-mediated pro-inflammatory response is also linked to a number of signaling pathways, including the NF-KB pathway (R. Li et al., 2021), which is a key component in the inflammatory response (Szatkowski et al., 2020). Studies have shown that IKBa is degraded once the NFKB pathway is activated, which increases the phosphorylation of p65, allowing p65 to translocate from the cytoplasm to the nucleus, resulting in inflammation (White, Lin, \& Hu, 2020). We found that Au not only inhibits the expression of $\mathrm{p}-\mathrm{I} \mathrm{KBa}$, but also inhibits $\mathrm{p} 65$ phosphorylation and regulates its nuclear translocation (Fig. 5D, E, F, and L), indicating that Au inhibits the NF-KB pathway. To investigate whether Au suppressed LPS-induced microglial activation via NF-KB signaling, we treated microglia with PDTC, an NF-kB pathway inhibitor. The results showed that Au or PDTC therapy lowered the protein levels of $\mathrm{p}-\mathrm{IkBa}$, p-p65, iNOS, and COX2. Furthermore, co-treatment with Au and PDTC significantly reduced the expression of p-IKBa, p-p65, and inflammatory markers (e.g., iNOS and COX2) (Fig. 5G-K). Fluorescence results showed that co-incubation with Au and PDTC resulted in significantly greater translocation of p65 to the nucleus compared with that observed in response to Au or PDTC incubation alone (Fig. $5 \mathrm{~L}$ ). Our findings 
showed that Au exerted anti-inflammatory properties by inactivating the NF-KB pathway, which was mediated by TLR4-MyD88.

Neuronal apoptosis is an important component of secondary injury (Mortezaee et al., 2018). Inhibition of neuronal apoptosis is thought to aid in neural rehabilitation and axon regeneration (Qi et al., 2021; Y. Wu et al., 2021). Therefore, development of strategies to reduce neuronal apoptosis in SCl has received greater interest. We used TUNEL staining to measure the level of cell apoptosis after acute $\mathrm{SCl}$, and the results showed that Au therapy dramatically reduced cell apoptosis (Fig. 6A and B). Furthermore, the protein level of NeuN (a neuron marker) was higher in the Au group than that in the SCl group (Fig. $6 \mathrm{C}$ and D). These results suggested that administration of $\mathrm{Au}$ after $\mathrm{SCl}$ inhibited neuronal apoptosis. Additionally, we found that, compared to the $\mathrm{SCl}$ group, the levels of the mitochondrial apoptosis-related proteins (BAX and Cleaved Caspase 3) rose in the $\mathrm{SCl}$ group, while the expression levels of the mitochondrial antiapoptotic protein Bcl-2 decreased; however, this result was reversed by Au treatment (Fig. 6E and F). Cleaved caspase 3 fluorescence staining results were consistent with those obtained using western blot (Fig. $6 \mathrm{G}$ and $\mathrm{H}$ ). We then used TBHP to activate PC12 cells in vitro to mimic the influence of oxidative stress on neuronal survival after SCl. CCK8 detection and live-dead labeling can both show that $\mathrm{Au}$ treatment clearly reversed TBHP-induced neuronal apoptosis (Fig. 7B, G and H). Additionally, after Au therapy, the amount of mitochondrial anti-apoptotic protein (Bcl-2) was partially restored (Fig. 7C-F). These results suggested that Au protected neurons from mitochondrial dysfunction-induced apoptosis.

Previous studies have shown that Au can ameliorate secondary injury by reducing inflammation and neuronal apoptosis caused after $\mathrm{SCl}$. The healing and expansion of neuronal axons is facilitated by successful control of secondary injury (Pelisch et al., 2020; C. Wang et al., 2021), and axon regeneration is thought to play a critical role in the recovery of motor function following SCl (C. Wang et al., 2019; Zhou et al., 2020). Therefore, we evaluated axonal recovery following SCl. Our findings showed that the expression of positive MAP2 axon structural proteins was higher, the region was more densely populated with axons, and axonal organization was more regular in the Au group than in the SCl group (Fig. 8B and C). We used immunofluorescence to stain GAP43 (a neurofilament marker), and showed that Au therapy increased GAP43-positive fibers in the lesion margin of SCl and extended toward the lesion center (Fig. 8D and E). When compared to the SCl group, MAP2 and GAP43 protein levels were partially restored in the Au group (Fig. 8F-H). These results indicated that treatment with Au stimulated regeneration of MAP2-positive axons and GAP43-positive neurofilaments by preventing secondary injury after SCI.

There were several limitations to our study. First, the long-term negative effects of Au injections are unknown. Second, Au is believed to exert a strong anti-inflammatory effect and to promote decreased expression of inflammatory mediators released by inflammatory cells via a variety of mechanisms, but additional mechanisms were not evaluated in this study. Furthermore, because several Au receptors have yet to be identified, it is uncertain whether the anti-inflammatory effects on different phenotypes of inflammatory cells and neuroprotective effects on neurons are mediated by different receptors.

\section{Conclusion}


In conclusion, our data showed that Au can promote recovery from SCl by blocking the TLR4/NF-KB pathway to regulate microglial polarization, thereby reducing inflammation and inhibiting neuronal apoptosis mediated by mitochondrial dysfunction.

\section{Abbreviations}

SCl: Spinal cord injury; Au: Aucubin; LPS: Lipopolysaccharide; TBHP: Tert-butyl hydroperoxide; PFA: paraformaldehyde; TLR4: the toll-like receptor 4; MyD88: myeloid differentiation protein-88; NF-KB: nuclear factor kappa B; IкBa: inhibitor kappa Ba; COX-2: cyclooxygenase-2; iNOS: inducible nitric oxide synthase; TNF-a: tumor necrosis factor-a; IL-1: Interleukin-1; IL-6: Interleukin-6; BBB: Basso, Beattie, and Bresnahan; CC-3: cleaved caspase-3.

\section{Declarations}

\section{Acknowledgements}

Thanks to the Public Platform Laboratory of the First Affiliated Hospital of Nanchang University for providing the experimental platform.

\section{Funding}

This work was sponsored by the "Double Thousand Plan" of Jiangxi Province, the National Natural Science Foundation of China (Grant No. 81860472), Nature Science Foundation of Jiangxi Province (Grant No. 20192ACBL21041; 20202BAB206044).

\section{Availability of data and materials}

The main original data are provided in this manuscript. Any other original data are available from the corresponding author when needed.

\section{Authors' contributions}

SX designed the project, performed most of the experiments, and wrote the manuscript. Numerous technical assistances were provided by NZ and QY. AL, WT and YZ helped with the collection of some experimental data. GY and SW participated in animal experiments. ZL and JL oversaw the project and provided funding. All authors read and approved the final manuscript.

\section{Ethics approval and consent to participate}

In animal experiments, all protocols are consistent with the guidelines of the Medical Animal Care \& Welfare Committee and were approved by the Laboratory Animal Ethics Committee of the First Affiliated Hospital of Nanchang University.

\section{Competing interests}


The authors confirm that they have no conflict of interest.

\section{Consent for publication}

Not applicable.

\section{References}

1. Álvarez Z, Kolberg-Edelbrock A, Sasselli I, Ortega J, Qiu R, Syrgiannis Z,.. . Stupp S. Bioactive scaffolds with enhanced supramolecular motion promote recovery from spinal cord injury. Sci (New York N Y ). 2021;374(6569):848-56. doi:10.1126/science.abh3602.

2. Bayan N, Yazdanpanah N, Rezaei N. (2021). Role of Toll-Like Receptor 4 in Diabetic Retinopathy. Pharmacol Res, 105960. doi:10.1016/j.phrs.2021.105960.

3. Boillée S. Local and remote interactions between macrophages and microglia in neurological conditions. Curr Opin Immunol. 2021;74:118-24. doi:10.1016/j.coi.2021.11.006.

4. Chen Z, Zhong D, Li G. The role of microglia in viral encephalitis: a review. J Neuroinflamm. 2019;16(1):76. doi:10.1186/s12974-019-1443-2.

5. Fan L, Liu C, Chen X, Zou Y, Zhou Z, Lin C,... Wang Q. Directing Induced Pluripotent Stem Cell Derived Neural Stem Cell Fate with a Three-Dimensional Biomimetic Hydrogel for Spinal Cord Injury Repair. ACS Appl Mater Interfaces. 2018;10(21):17742-55. doi:10.1021/acsami.8b05293.

6. Gao F, Li H, Feng Y, Tian W, Cao R, Fu K. Aucubin ameliorates the LPS-induced inflammatory response in bovine endometrial epithelial cells by inhibiting NF-KB and activating the Keap1/Nrf2 signalling pathway. Reprod Domest animals = Zuchthygiene. 2021;56(7):972-82. doi:10.1111/rda.13939.

7. Gaojian T, Dingfei Q, Linwei L, Xiaowei W, Zheng Z, Wei L,... . Jian C. Parthenolide promotes the repair of spinal cord injury by modulating M1/M2 polarization via the NF-KB and STAT $1 / 3$ signaling pathway. Cell death discovery. 2020;6(1):97. doi:10.1038/s41420-020-00333-8.

8. Giraldo E, Nebot V, Đorđević S, Requejo-Aguilar R, Alastrue-Agudo A, Zagorodko O,.. . MorenoManzano $\mathrm{V}$. A rationally designed self-immolative linker enhances the synergism between a polymerrock inhibitor conjugate and neural progenitor cells in the treatment of spinal cord injury. Biomaterials. 2021;276:121052. doi:10.1016/j.biomaterials.2021.121052.

9. Gong Z, Wang C, Ni L, Ying L, Shu J, Wang J,.. . Liang C. (2020). An injectable recombinant human milk fat globule-epidermal growth factor 8-loaded copolymer system for spinal cord injury reduces inflammation through NF-kappaB and neuronal cell death. Cytotherapy, 22(4), 193-203. Retrieved from https://www.ncbi.nlm.nih.gov/pubmed/32173261. doi:10.1016/j.jcyt.2020.01.016.

10. Hellenbrand D, Quinn C, Piper Z, Morehouse C, Fixel J, Hanna A. Inflammation after spinal cord injury: a review of the critical timeline of signaling cues and cellular infiltration. J Neuroinflamm. 2021;18(1):284. doi:10.1186/s12974-021-02337-2. 
11. Huang KT, Lu Y. Traumatic Spinal Cord Disorders: Current Topics and Future Directions. Semin Neurol. 2021;41(3):247-55. doi:10.1055/s-0041-1725125.

12. Huang W, Lin M, Yang C, Wang F, Zhang M, Gao J, Yu X. (2021). Rat Bone Mesenchymal Stem CellDerived Exosomes Loaded with miR-494 Promoting Neurofilament Regeneration and Behavioral Function Recovery after Spinal Cord Injury. Oxidative medicine and cellular longevity, 2021, 1634917. doi:10.1155/2021/1634917.

13. Jeong J, Lee H, Han M, Kim G, Kim W, Choi Y. Anti-inflammatory effects of genistein via suppression of the toll-like receptor 4-mediated signaling pathway in lipopolysaccharide-stimulated BV2 microglia. Chemico-Biol Interact. 2014;212:30-9. doi:10.1016/j.cbi.2014.01.012.

14. Jiang S, Baba K, Okuno T, Kinoshita M, Choong C, Hayakawa H,.. . Mochizuki H. Go-sha-jinki-Gan Alleviates Inflammation in Neurological Disorders via p38-TNF Signaling in the Central Nervous System. Neurotherapeutics: the journal of the American Society for Experimental NeuroTherapeutics. 2021;18(1):460-73. doi:10.1007/s13311-020-00948-w.

15. Jin X, Liu M, Zhang D, Zhong X, Du K, Qian P,.. . Wei M. Natural products as a potential modulator of microglial polarization in neurodegenerative diseases. Pharmacol Res. 2019;145:104253. doi:10.1016/j.phrs.2019.104253.

16. Kim Y, Sim U, Shin Y, Kim Kwon Y. Aucubin promotes neurite outgrowth in neural stem cells and axonal regeneration in sciatic nerves. Experimental Neurobiol. 2014;23(3):238-45. doi:10.5607/en.2014.23.3.238.

17. Koushki K, Shahbaz S, Mashayekhi K, Sadeghi M, Zayeri Z, Taba M,... Sahebkar A. Anti-inflammatory Action of Statins in Cardiovascular Disease: the Role of Inflammasome and Toll-Like Receptor Pathways. Clin Rev Allergy Immunol. 2021;60(2):175-99. doi:10.1007/s12016-020-08791-9.

18. Li R, Zhou Y, Zhang S, Li J, Zheng Y, Fan X. The natural (poly)phenols as modulators of microglia polarization via TLR4/NF-kB pathway exert anti-inflammatory activity in ischemic stroke. Eur J Pharmacol. 2021;914:174660. doi:10.1016/j.ejphar.2021.174660.

19. Li Y, Jiang Q, Wang L. Appetite Regulation of TLR4-Induced Inflammatory Signaling. Front Endocrinol. 2021;12:777997. doi:10.3389/fendo.2021.777997.

20. Lin J, Pan X, Huang C, Gu M, Chen X, Zheng X,... Wang X. Dual regulation of microglia and neurons by Astragaloside IV-mediated mTORC1 suppression promotes functional recovery after acute spinal cord injury. J Cell Mol Med. 2020;24(1):671-85. doi:10.1111/jcmm.14776.

21. Liu Z, Yao X, Sun B, Jiang W, Liao C, Dai X,.. . Ding R. Pretreatment with kaempferol attenuates microglia-mediate neuroinflammation by inhibiting MAPKs-NF-KB signaling pathway and pyroptosis after secondary spinal cord injury. Free Radic Biol Med. 2021;168:142-54. doi:10.1016/j.freeradbiomed.2021.03.037.

22. Luo Z, Peng W, Xu Y, Xie Y, Liu Y, Lu H,.. . Hu J. Exosomal OTULIN from M2 macrophages promotes the recovery of spinal cord injuries via stimulating Wnt/ $\beta$-catenin pathway-mediated vascular regeneration. Acta Biomater. 2021. doi:10.1016/j.actbio.2021.09.026. 
23. Ma S, Chen Y, Zhang J, Shen L, Wang R, Zhou J,.. . Lü H. Adoptive transfer of M2 macrophages promotes locomotor recovery in adult rats after spinal cord injury. Brain Behav Immun. 2015;45:15770. doi:10.1016/j.bbi.2014.11.007.

24. Macks $C$, Jeong $D$, Lee J. Local delivery of RhoA siRNA by PgP nanocarrier reduces inflammatory response and improves neuronal cell survival in a rat TBI model. Nanomedicine: Nanatechnol biology Med. 2021;32:102343. doi:10.1016/j.nano.2020.102343.

25. Mortezaee K, Khanlarkhani N, Beyer C, Zendedel A. Inflammasome: Its role in traumatic brain and spinal cord injury. J Cell Physiol. 2018;233(7):5160-9. doi:10.1002/jcp.26287.

26. Moujalled D, Strasser A, Liddell J. Molecular mechanisms of cell death in neurological diseases. Cell Death Differ. 2021;28(7):2029-44. doi:10.1038/s41418-021-00814-y.

27. Pan Q, Lin F, Liu N, Chen R. The role of aquaporin 4 (AQP4) in spinal cord injury. Biomed pharmacotherapy $=$ Biomedecine pharmacotherapie. 2022;145:112384.

doi:10.1016/j.biopha.2021.112384.

28. Pang Q-M, Chen S-Y, Xu Q-J, Fu S-P, Yang Y-C, Zou W-H,... Zhang T. (2021). Neuroinflammation and Scarring After Spinal Cord Injury: Therapeutic Roles of MSCs on Inflammation and Glial Scar. Frontiers in Immunology, 12(5158). Retrieved from https://www.frontiersin.org/article/10.3389/fimmu.2021.751021. doi:10.3389/fimmu.2021.751021.

29. Pang Q, Chen S, Xu Q, Fu S, Yang Y, Zou W,... Zhang T. Neuroinflammation and Scarring After Spinal Cord Injury: Therapeutic Roles of MSCs on Inflammation and Glial Scar. Front Immunol. 2021;12:751021. doi:10.3389/fimmu.2021.751021.

30. Park J, Zhang Y, Saito E, Gurczynski S, Moore B, Cummings B,.. . Shea L. Intravascular innate immune cells reprogrammed via intravenous nanoparticles to promote functional recovery after spinal cord injury. Proc Natl Acad Sci USA. 2019;116(30):14947-54. doi:10.1073/pnas.1820276116.

31. Pelisch N, Rosas Almanza J, Stehlik K, Aperi B, Kroner A. CCL3 contributes to secondary damage after spinal cord injury. J Neuroinflamm. 2020;17(1):362. doi:10.1186/s12974-020-02037-3.

32. Qi J, Wang T, Zhang Z, Yin Z, Liu Y, Ma L,... . Han G. Circ-Ctnnb1 regulates neuronal injury in spinal cord injury through Wnt/ß-catenin signaling pathway. Dev Neurosci. 2021. doi:10.1159/000521172.

33. Ramer $L$, Ramer $M$, Bradbury E. Restoring function after spinal cord injury: towards clinical translation of experimental strategies. Lancet Neurol. 2014;13(12):1241-56. doi:10.1016/s14744422(14)70144-9.

34. Rivers C, Fallah N, Noonan V, Whitehurst D, Schwartz C, Finkelstein J,... Noreau L. Health Conditions: Effect on Function, Health-Related Quality of Life, and Life Satisfaction After Traumatic Spinal Cord Injury. A Prospective Observational Registry Cohort Study. Arch Phys Med Rehabil. 2018;99(3):44351. doi:10.1016/j.apmr.2017.06.012.

35. Ronaldson P, Davis T. Regulation of blood-brain barrier integrity by microglia in health and disease: A therapeutic opportunity. J Cereb blood flow metabolism: official J Int Soc Cereb Blood Flow Metabolism. 2020;40:6-24. doi:10.1177/0271678x20951995. 
36. Sámano C, Nistri A. Mechanism of Neuroprotection Against Experimental Spinal Cord Injury by Riluzole or Methylprednisolone. Neurochem Res. 2019;44(1):200-13. doi:10.1007/s11064-017-24596.

37. Saunders L, Clarke A, Tate D, Forchheimer M, Krause J. Lifetime prevalence of chronic health conditions among persons with spinal cord injury. Arch Phys Med Rehabil. 2015;96(4):673-9. doi:10.1016/j.apmr.2014.11.019.

38. Scott M, Bedi S, Olson S, Sears C, Cox C. (2021). Microglia as therapeutic targets after neurological injury: strategy for cell therapy. Expert Opin Ther Targets, 1-16.

doi:10.1080/14728222.2021.1934447.

39. Shen H, Xu B, Yang C, Xue W, You Z, Wu X,... Dai J. A DAMP-scavenging, IL-10-releasing hydrogel promotes neural regeneration and motor function recovery after spinal cord injury. Biomaterials. 2021;280:121279. doi:10.1016/j.biomaterials.2021.121279.

40. Szatkowski P, Krzysciak W, Mach T, Owczarek D, Brzozowski B, Szczeklik K. (2020). Nuclear factor-kB - importance, induction of inflammation, and effects of pharmacological modulators in Crohn's disease. J Physiol pharmacology: official J Pol Physiological Soc, 71(4). doi:10.26402/jpp.2020.4.01.

41. Tran A, Warren P, Silver J. The Biology of Regeneration Failure and Success After Spinal Cord Injury. Physiol Rev. 2018;98(2):881-917. doi:10.1152/physrev.00017.2017.

42. Walsh C, Wychowaniec J, Brougham D, Dooley D. (2021). Functional hydrogels as therapeutic tools for spinal cord injury: New perspectives on immunopharmacological interventions. Pharmacol Ther, 108043. doi:10.1016/j.pharmthera.2021.108043.

43. Wan F, Zang S, Yu G, Xiao H, Wang J, Tang J. Ginkgolide B Suppresses Methamphetamine-Induced Microglial Activation Through TLR4-NF-kB Signaling Pathway in BV2 Cells. Neurochem Res. 2017;42(10):2881-91. doi:10.1007/s11064-017-2309-6.

44. Wang C, Gong Z, Huang X, Wang J, Xia K, Ying L,.. . Chen Q. An injectable heparin-Laponite hydrogel bridge FGF4 for spinal cord injury by stabilizing microtubule and improving mitochondrial function. Theranostics. 2019;9(23):7016-32. doi:10.7150/thno.37601.

45. Wang C, Wang M, Xia K, Wang J, Cheng F, Shi K,.. . Chen Q. A bioactive injectable self-healing antiinflammatory hydrogel with ultralong extracellular vesicles release synergistically enhances motor functional recovery of spinal cord injury. Bioactive Mater. 2021;6(8):2523-34. doi:10.1016/j.bioactmat.2021.01.029.

46. Wang C, Wang Q, Lou Y, Xu J, Feng Z, Chen Y,... Zhang X. Salidroside attenuates neuroinflammation and improves functional recovery after spinal cord injury through microglia polarization regulation. $J$ Cell Mol Med. 2018;22(2):1148-66. doi:10.1111/jcmm.13368.

47. Wang H, Zhou X, Wu L, Liu G, Xu W, Zhang X,... Li W. Aucubin alleviates oxidative stress and inflammation via Nrf2-mediated signaling activity in experimental traumatic brain injury. J Neuroinflamm. 2020;17(1):188. doi:10.1186/s12974-020-01863-9. 
48. Wang L, Zhang P, Li C, Xu F, Chen J. Rosa roxburghiiA polysaccharide from Tratt fruit attenuates high-fat diet-induced intestinal barrier dysfunction and inflammation in mice by modulating the gut microbiota. Food Funct. 2021. doi:10.1039/d1fo03190b.

49. White S, Lin L, Hu K. (2020). NF-KB and tPA Signaling in Kidney and Other Diseases. Cells, 9(6). doi:10.3390/cells9061348.

50. Williams P, Marincu B, Sorbara C, Mahler C, Schumacher A, Griesbeck O,.. . Misgeld T. A recoverable state of axon injury persists for hours after spinal cord contusion in vivo. Nat Commun. 2014;5:5683. doi:10.1038/ncomms6683.

51. Wu C, Chen S, Zhou T, Wu K, Qiao Z, Zhang Y,.. . Fan H. Antioxidative and Conductive NanoparticlesEmbedded Cell Niche for Neural Differentiation and Spinal Cord Injury Repair. ACS Appl Mater Interfaces. 2021. doi:10.1021/acsami.1c14679.

52. Wu Y, Xiong J, He Z, Yuan Y, Wang B, Xu J,.. . Xiao J. Metformin promotes microglial cells to facilitate myelin debris clearance and accelerate nerve repairment after spinal cord injury. Acta Pharmacol Sin. 2021. doi:10.1038/s41401-021-00759-5.

53. Xiao S, Wang C, Yang Q, Xu H, Lu J, Xu K. Rea regulates microglial polarization and attenuates neuronal apoptosis via inhibition of the NF-KB and MAPK signalings for spinal cord injury repair. $J$ Cell Mol Med. 2021;25(3):1371-82. doi:10.1111/jcmm.16220.

54. Yang Z, Wu Q, Xiao Y, Duan M, Liu C, Yuan Y,.. . Tang Q. (2018). Aucubin Protects against Myocardial Infarction-Induced Cardiac Remodeling via nNOS/NO-Regulated Oxidative Stress. Oxidative medicine and cellular longevity, 2018, 4327901. doi:10.1155/2018/4327901.

55. Yuan X, Yuan W, Ding L, Shi M, Luo L, Wan Y,.. . Deng D. Cell-adaptable dynamic hydrogel reinforced with stem cells improves the functional repair of spinal cord injury by alleviating neuroinflammation. Biomaterials. 2021;279:121190. doi:10.1016/j.biomaterials.2021.121190.

56. Zeng H, Liu N, Yang Y, Xing H, Liu X, Li F,.. . Zhou M. Lentivirus-mediated downregulation of asynuclein reduces neuroinflammation and promotes functional recovery in rats with spinal cord injury. J Neuroinflamm. 2019;16(1):283. doi:10.1186/s12974-019-1658-2.

57. Zhang J, Li Y, Xiong J, Xu H, Xiang G, Fan M,.. . Xiao J. Delivery of pOXR1 through an injectable liposomal nanoparticle enhances spinal cord injury regeneration by alleviating oxidative stress. Bioactive Mater. 2021;6(10):3177-91. doi:10.1016/j.bioactmat.2021.03.001.

58. Zhang S, Feng Z, Gao W, Duan Y, Fan G, Geng X,.. . Peng C. Aucubin Attenuates Liver IschemiaReperfusion Injury by Inhibiting the HMGB1/TLR-4/NF-KB Signaling Pathway, Oxidative Stress, and Apoptosis. Front Pharmacol. 2020;11:544124. doi:10.3389/fphar.2020.544124.

59. Zhao T, Yan W, Xu K, Qi Y, Dai X, Shi Z. Combined treatment with platelet-rich plasma and brainderived neurotrophic factor-overexpressing bone marrow stromal cells supports axonal remyelination in a rat spinal cord hemi-section model. Cytotherapy. 2013;15(7):792-804. doi:10.1016/j.jcyt.2013.04.004.

60. Zheng Y, Qi S, Wu F, Hu J, Zhong R, Hua C,.. . Quan R. Chinese Herbal Medicine in Treatment of Spinal Cord Injury: A Systematic Review and Meta-Analysis of Randomized Controlled Trials. Am J Chin 
Med. 2020;48(7):1593-616. doi:10.1142/s0192415x20500792.

61. Zhou J, Li Z, Wu T, Zhao Q, Zhao Q, Cao Y. LncGBP9/miR-34a axis drives macrophages toward a phenotype conducive for spinal cord injury repair via STAT1/STAT6 and SOCS3. J Neuroinflamm. 2020;17(1):134. doi:10.1186/s12974-020-01805-5.

\section{Figures}
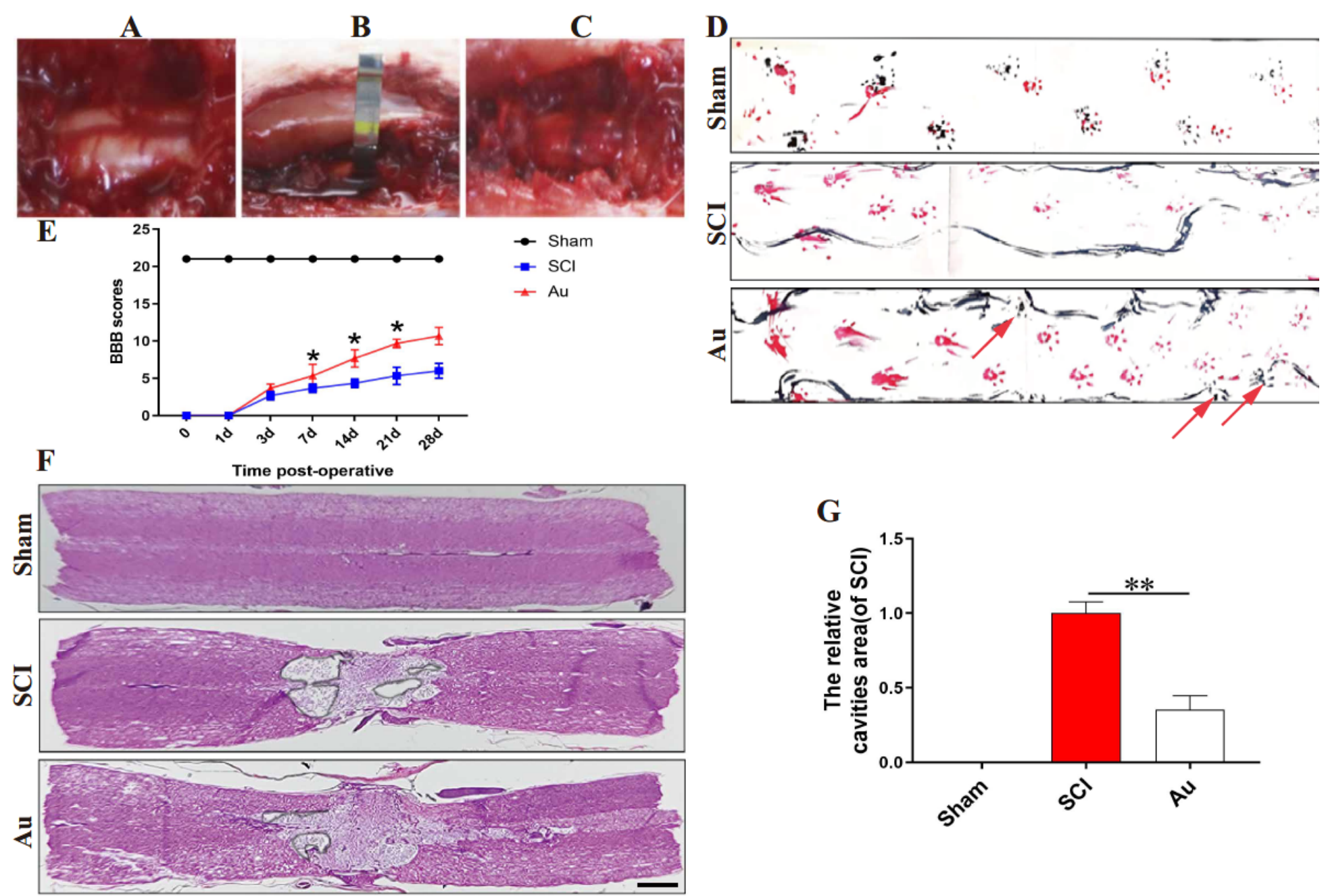

Figure 1

Au improved motor function after $\mathrm{SCl}$ and reduced cavity area. (A) The spinal cord of a rat that has been exposed. (B) A vascular clip is used to compress the exposed spinal cord. (C) The site of the crushing. (D, E) The behavior assay of footprint assay and BBB scores at 28 days postinjury (red arrow pointed the posterior limb prints). (F, G) H\&E staining was used to calculate the extent of the lesion cavity in each group at 28 days postinjury (scale bar $=1000 \mu \mathrm{m}$ ). $\mathrm{N} \geq 3$ per group for separate experiments. ${ }^{\star *} \mathrm{P}$ and $* \star * \mathrm{P}$ indicate Au group versus $\mathrm{SCl}$ group. ${ }^{*} \mathrm{P}<0.05$ and ${ }^{*} \mathrm{P}<0.01$. 


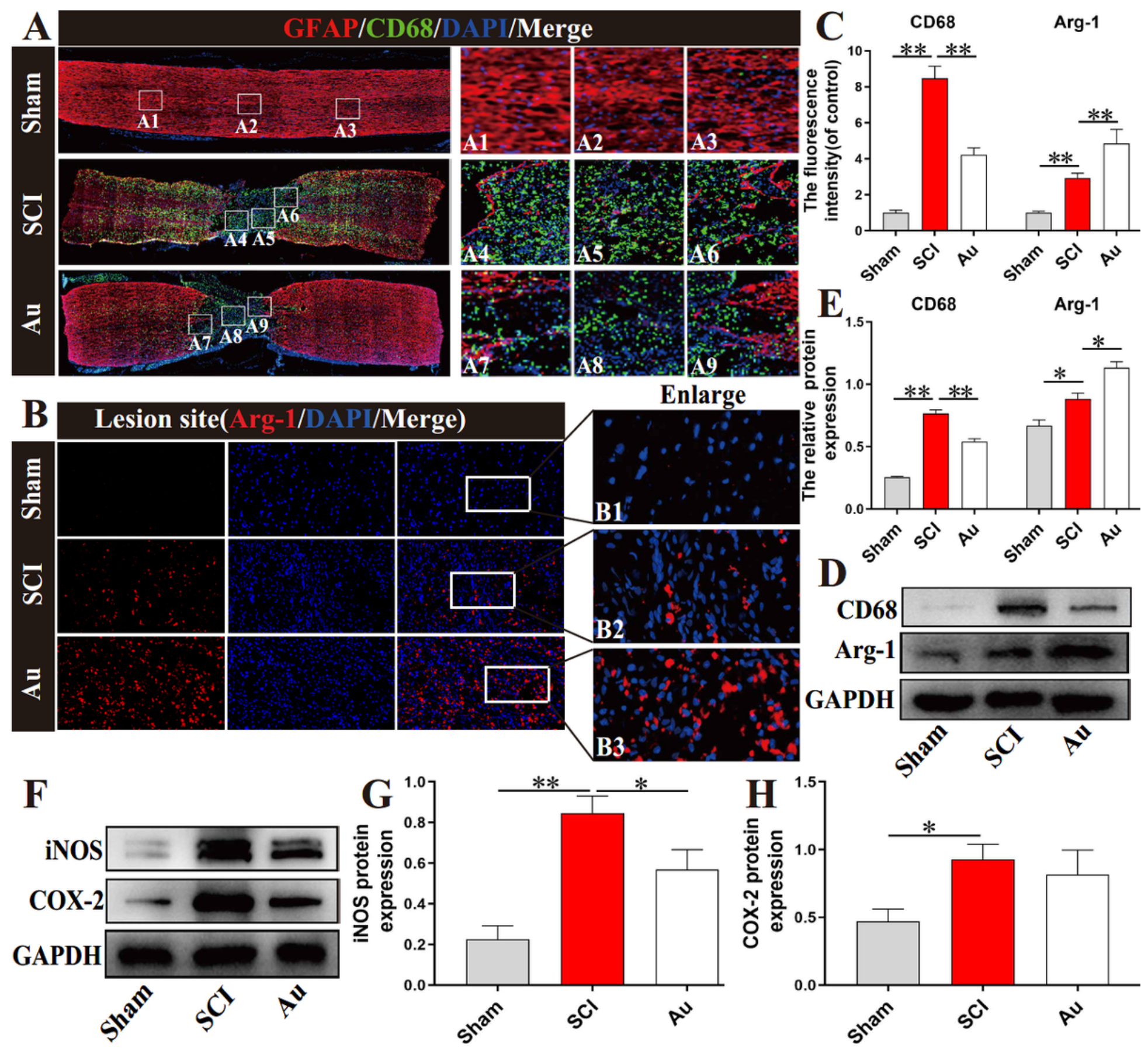

Figure 2

Au treatment suppresses the inflammatory response after SCl. (A, C) CD68 (green)/GFAP (red) double fluorescence labeling and quantitative examination of spinal cord tissue sections in each group at 3 days postinjury (scale bar=1000 $\mu \mathrm{m}$ ). A1, A4 and A7 are the enlarged views of the solid white box area from the rostral area. A2, A5 and A8 are the enlarged views of the solid white box area from the lesion area. A3, A6 and $A 9$ are the enlarged views of the solid white box area from the caudal area. (B, C) Representative images containing Arg-1 staining and quantification analysis in each group at 3 days after $\mathrm{SCl}$ (scale bar $=100 \mu \mathrm{m})$. B1, B2 and B3 are the enlarged figures of the solid white box area. (D, E) CD68 and Arg-1 proteins were detected and quantified in each group at 3 days postinjury. $(F, G, H)$ iNOS and COX-2 
proteins were detected and quantified in each group at 3 days postinjury. $\mathrm{N} \geq 3$ per group for separate experiments. ${ }^{*} \mathrm{P}<0.05$ and ${ }^{* *} \mathrm{P}<0.01$.
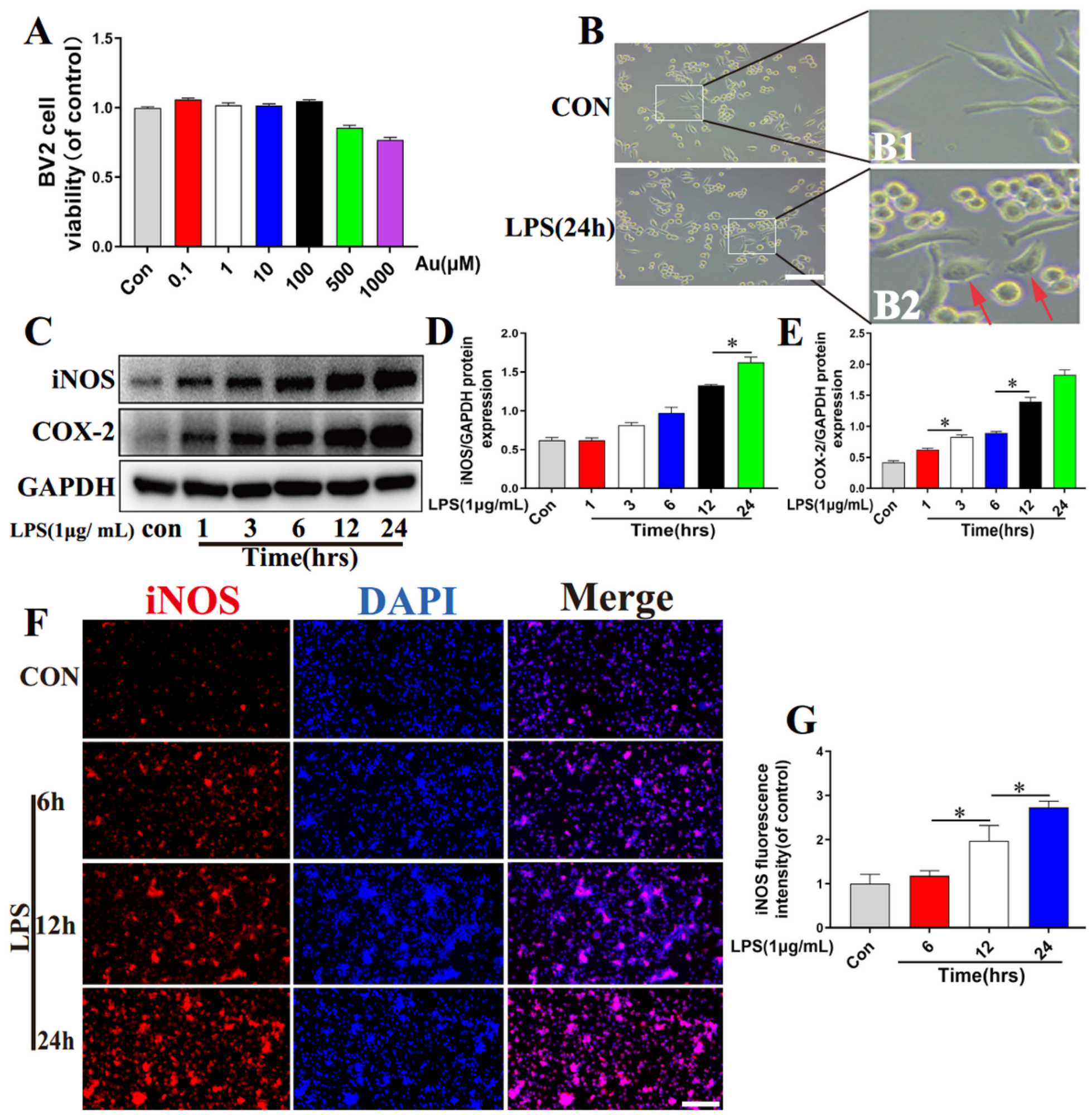

Figure 3

M1 microglia polarization was triggered by LPS in a time-dependent manner. (A) The CCK-8 test was applied to assess the viability of BV2 cells treated with Au at various doses for 24 hours. (B) Morphological change of BV2 cells (scale bar $=100 \mu \mathrm{m}$ ). B1 and B2 are the enlarged figures of the solid 
white box area (red arrow pointed the amoebic change). (C, D, E) iNOS and COX-2 proteins were detected and quantified in each group of microglia. $(F, G)$ iNOS level was measured by immunofluorescence labeling and quantification examination in each group of microglia (scale bar $=200 \mu \mathrm{m}$ ). $\mathrm{N} \geq 3$ per group for separate experiments. ${ }^{*} \mathrm{P}<0.05$.

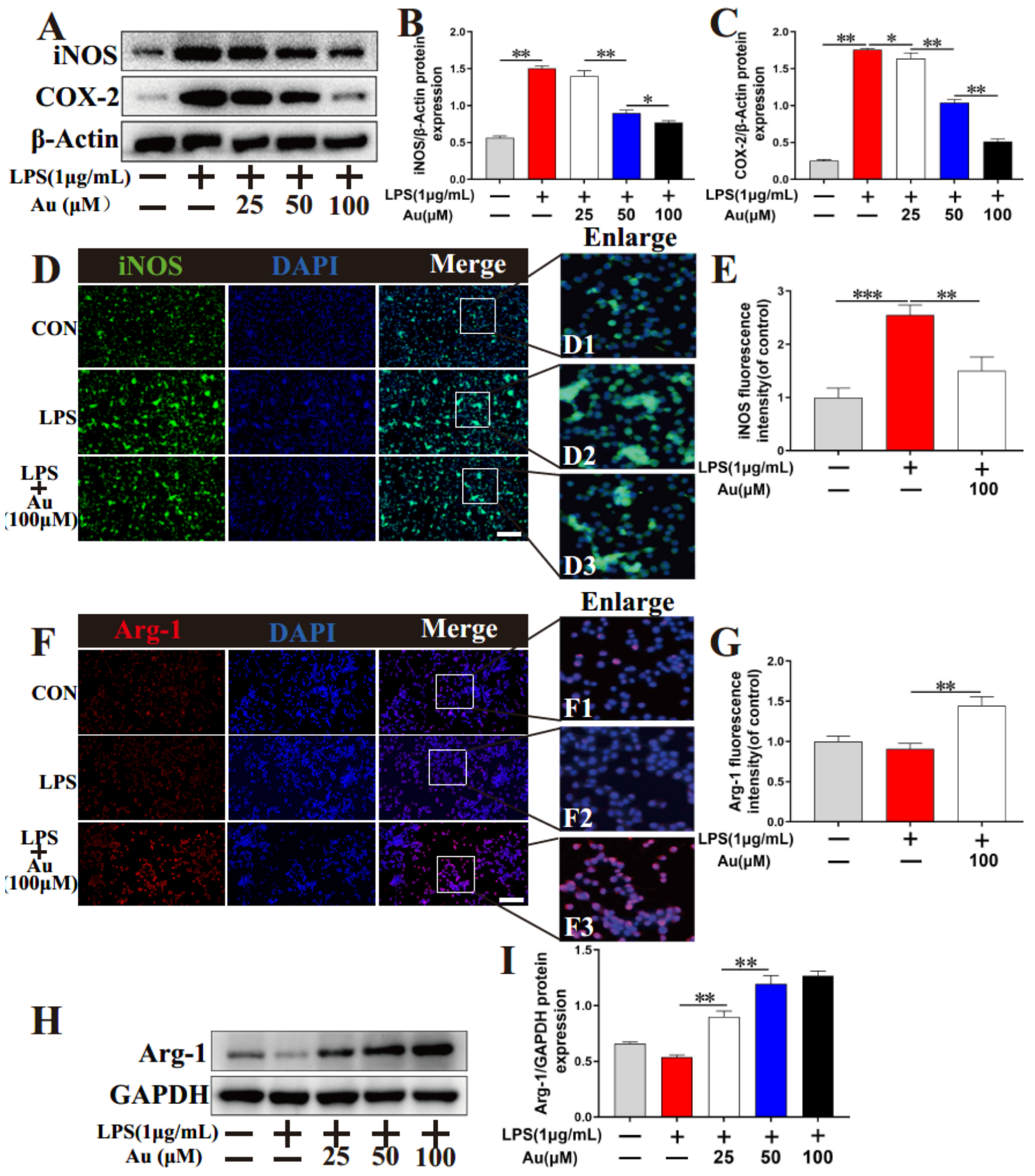

Figure 4 
Au promotes M2/M1 cell polarization ratio in microglia. (A, B, C) iNOS and COX-2 proteins were detected and quantified in microglia. (D, E) Immunofluorescence staining and quantitative data for iNOS in each

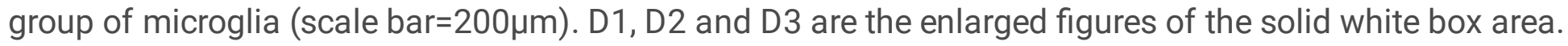
$(F, G)$ Immunofluorescence staining and quantification examination revealed the expression levels of Arg1 in each group of microglia (scale bar $=200 \mu \mathrm{m}$ ). F1, F2 and F3 are the enlarged figures of the solid white box area. $(H, I)$ Arg-1 protein was detected and quantified in each group of BV2 cells. $N \geq 3$ per group for separate experiments. ${ }^{*} \mathrm{P}<0.05,{ }^{*} \mathrm{P}<0.01$ and $* \star * \mathrm{P}<0.001$.

A
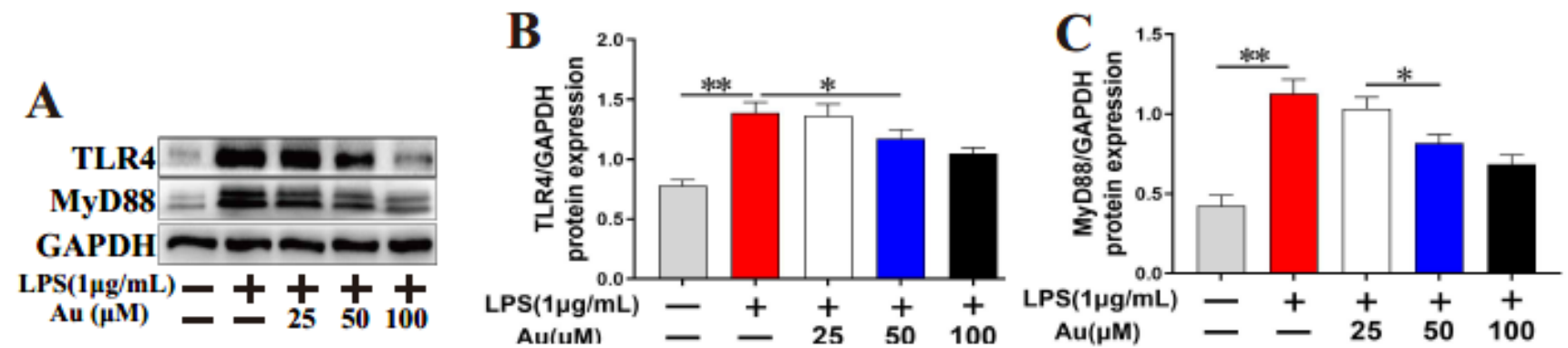

D

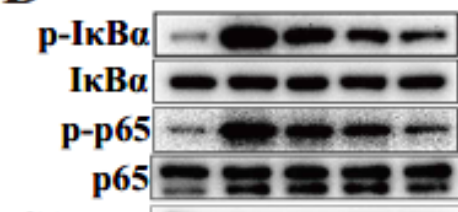

GAPDH

$\mathrm{LPS}(1 \mu \mathrm{g} / \mathrm{mL})-++t+$ Au $(\mu \mathrm{M}) \quad-\quad 25 \quad 50100$
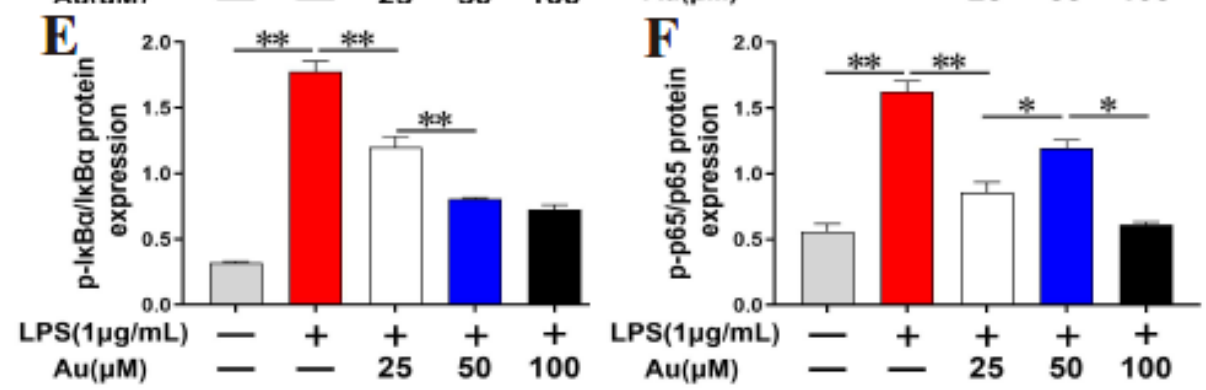

G

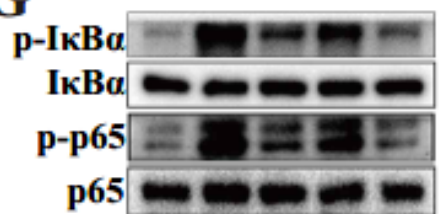

GAPDH

$\operatorname{LPS}(1 \mu \mathrm{g} / \mathrm{mL})-+++4$ $\mathrm{Au}(100 \mu \mathrm{M} / \mathrm{mL})$
PDTC $(10 \mu \mathrm{M})-\leq \pm+千$

K ${ }^{2.0} 7$ inOS/GAPDH COX-2/GAPDH
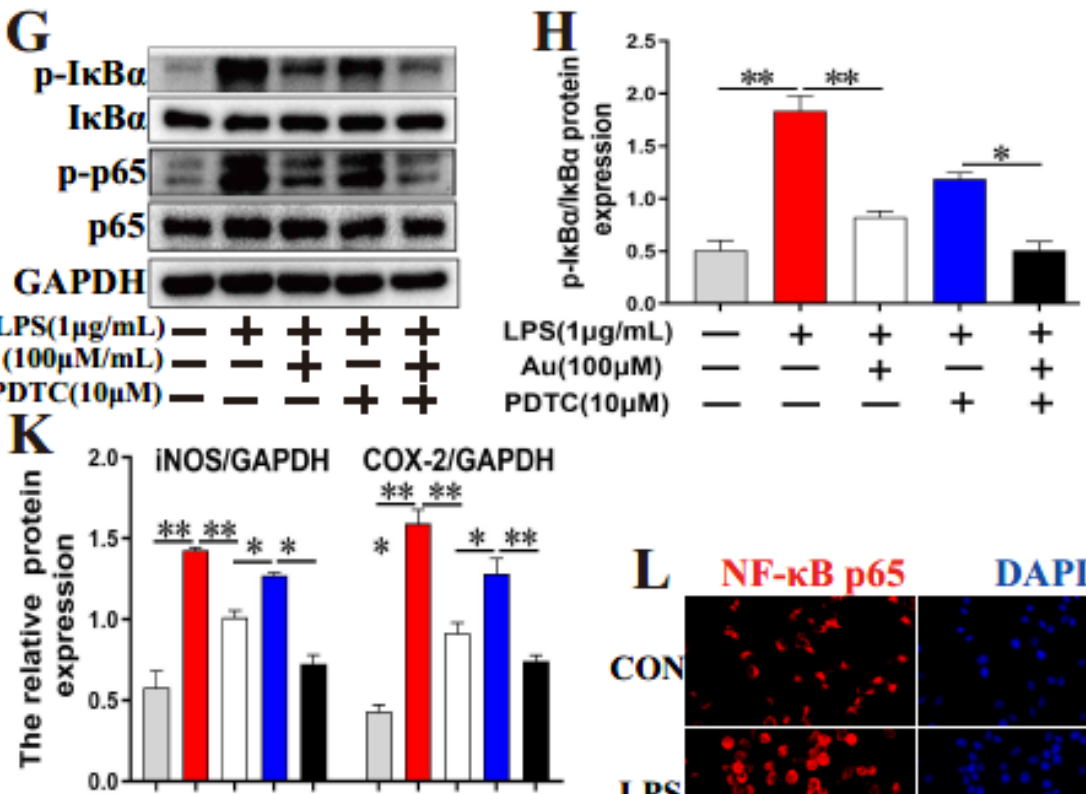

$\mathrm{LPS}(1 \mu \mathrm{g} / \mathrm{mL})-++++-++++$ $\mathrm{Au}(\mu \mathrm{M}) \quad--+-+--++$ $\operatorname{PDTC}(10 \mu \mathrm{M})---++--++$

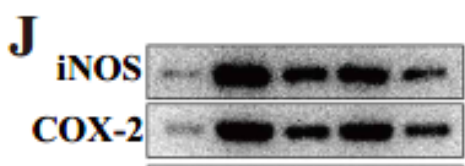

GAPDH

$\operatorname{LPS}(1 \mu \mathrm{g} / \mathrm{mL})-++t+$ $\mathrm{Au}(100 \mu \mathrm{M} / \mathrm{mL})= \pm+ \pm$

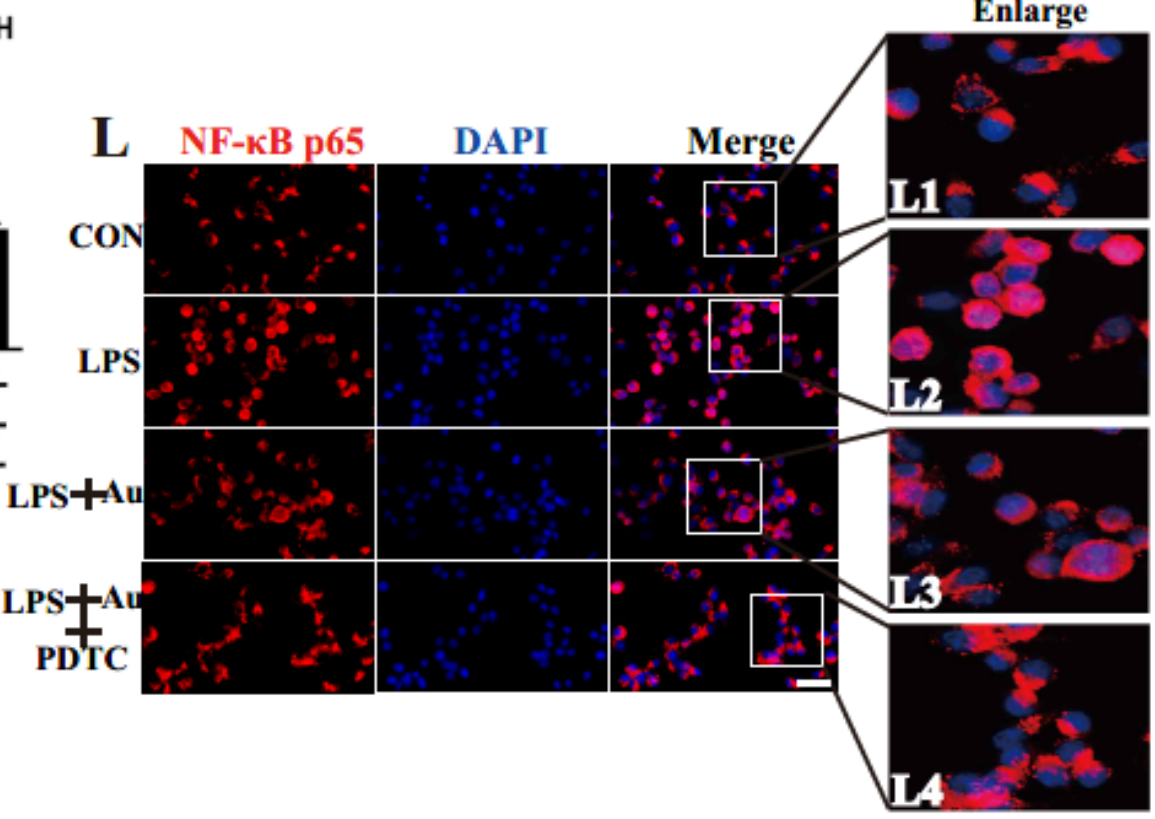

Page $22 / 26$ 


\section{Figure 5}

Au treatment suppresses inflammatory response via TLR4/NF-KB pathways in vitro. (A, B, C) TLR4 and MyD88 proteins were detected and quantified in each group of microglia. (D, E, F, G, H, I) Protein expressions IKBa, p-IKBa, p65 and p-p65 in each group of microglia. $(\mathrm{J}, \mathrm{K})$ iNOS and COX-2 proteins were detected and quantified in each group of microglia. (L) The translocation of the NF-KB p65 was determined by immunofluorescence staining in each group of microglia. (scale bar=50 $\mu \mathrm{m}$ ). L1, L2 and L3 are the enlarged figures of the solid white box area. $\mathrm{N} \geq 3$ per group for separate experiments. ${ }^{*} \mathrm{P}<0.05$ and $* * P<0.01$.

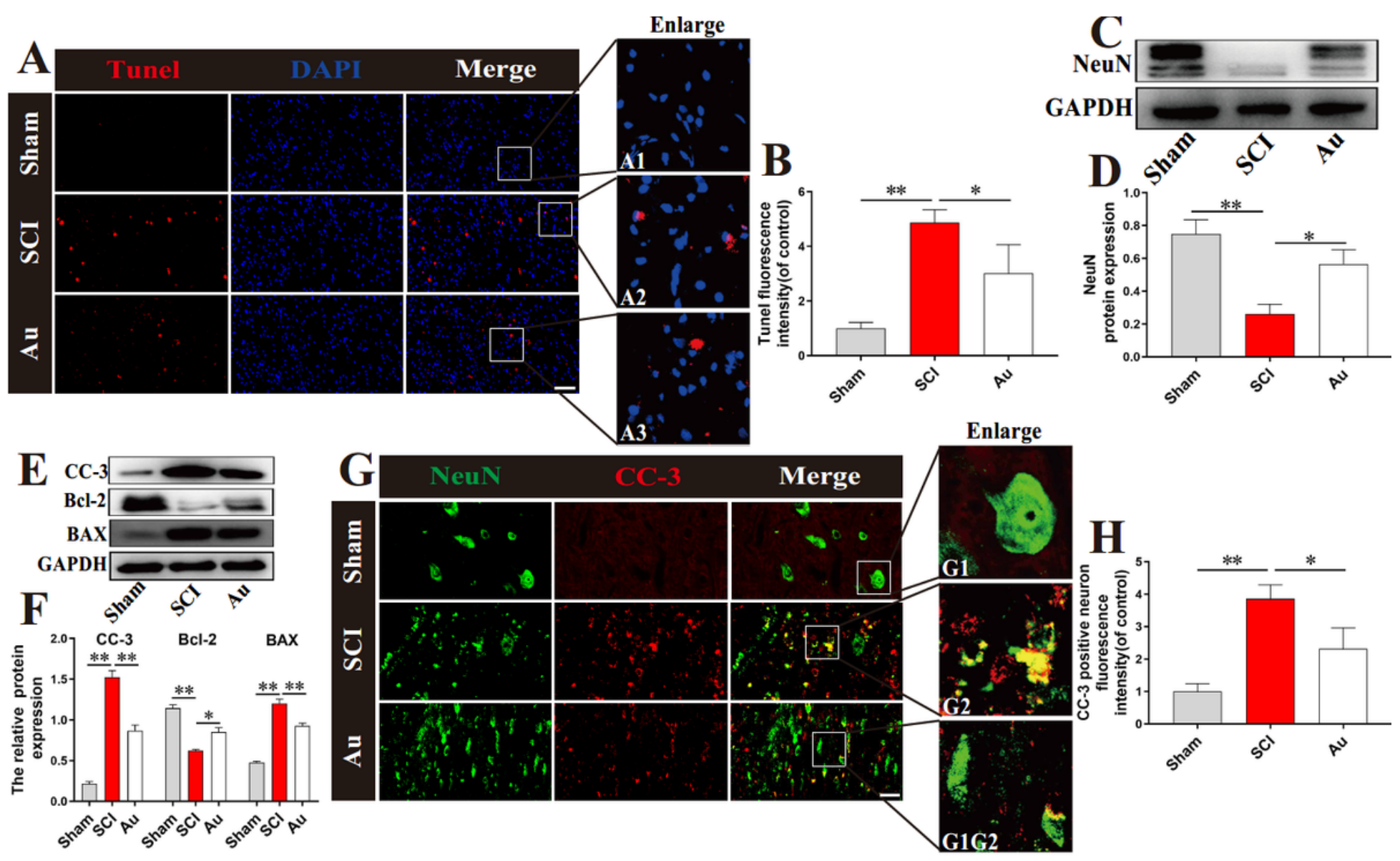

Figure 6

Pretreatment with Au prevents apoptosis in spinal neurons after SCl. (A, B) Tunel staining and quantitative analysis were used to detect neuronal apoptosis in each group at 3 days postinjury (scale bar $=100 \mu \mathrm{m}) . A 1, A 2$ and A3 are the enlarged figures of the solid white box area. (C, D) NeuN protein level were detected and quantified in each group at 3 days postinjury. (E, F) Apoptosis-related levels (CC-3, Bcl2 and $B A X)$ were detected and quantified in each group at 3 days postinjury. $(G, H)$ Representative images and quantification analysis of CC-3 (red) and NeuN (green) fluorescence staining in each group at 3 days

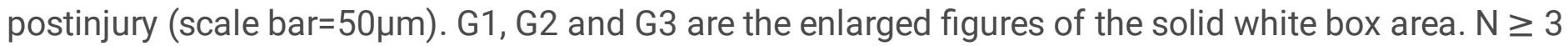
per group for separate experiments. ${ }^{*} \mathrm{P}<0.05$ and ${ }^{*} \mathrm{P}<0.01$. 


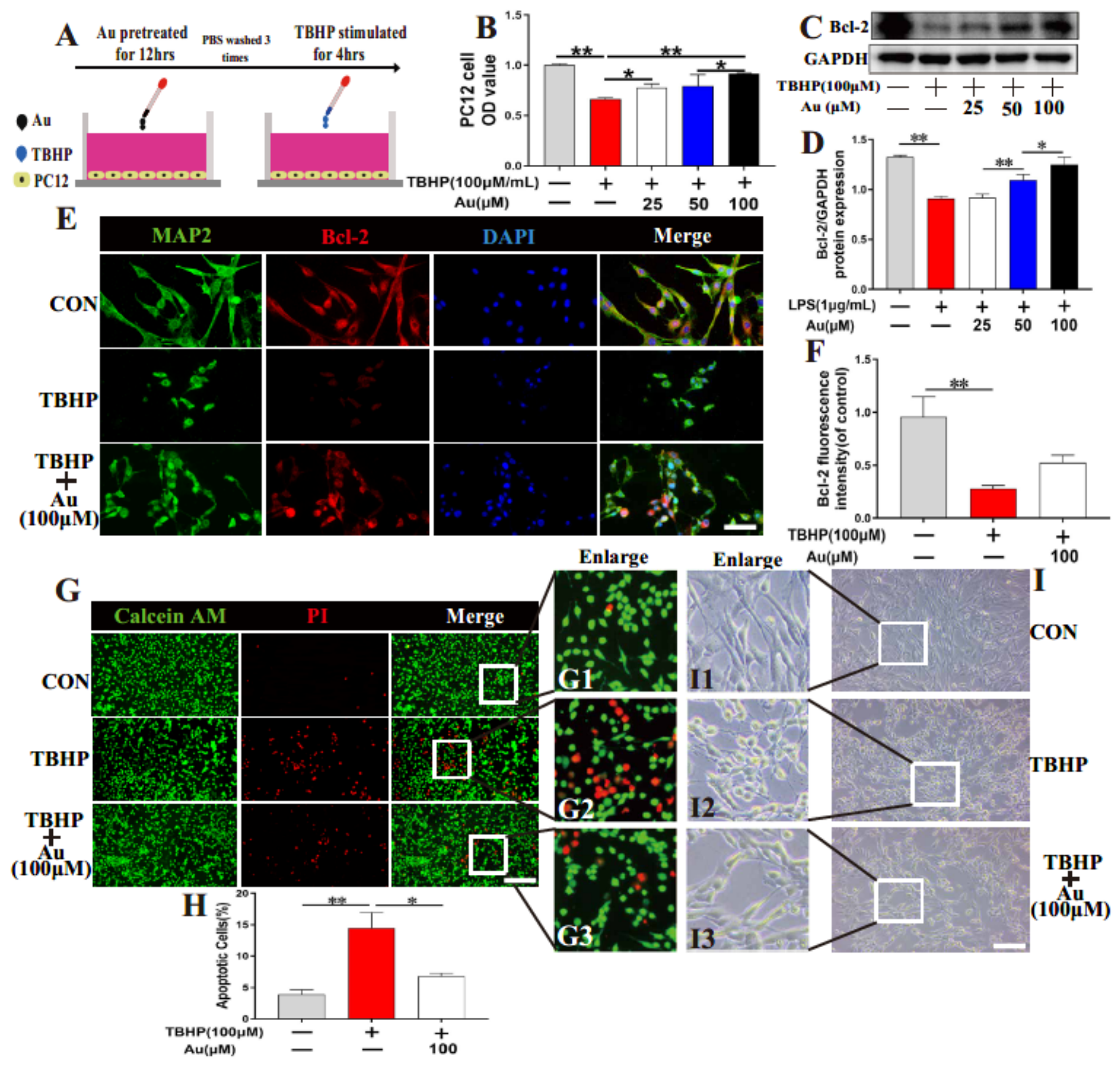

Figure 7

Au reduces neuronal apoptosis induced by TBHP. (A) Treatments for PC12 cells are depicted in this diagram. PC12 cells were pretreated for 12 hours with Au, then washed three times before being stimulated with TBHP for 4 hours. (B) The effect of Au pretreatment on the viability of PC12 cells was evaluated by CCK-8 test in each group. (C, D) Representative western blotting images and quantification showing Bcl-2 protein levels in each group of PC12 cells. (E, F) MAP2 and Bcl-2 immunolabeling and

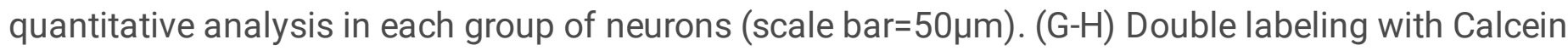
$\mathrm{AM} / \mathrm{PI}$ and quantitative examination of neuronal apoptosis in each group of PC12 cells (scale bar=200 $\mu \mathrm{m})$. G1, G2 and G3 are the enlarged figures of the solid white box area. (I) Morphological change 
of PC12 cells in each group (scale bar $=200 \mu \mathrm{m}$ ). 11,12 and 13 are the enlarged figures of the solid white box area. $N \geq 3$ per group for separate experiments. ${ }^{*} P<0.05,{ }^{\star *} P<0.01$ and ${ }^{* \star *} \mathrm{P}<0.001$.

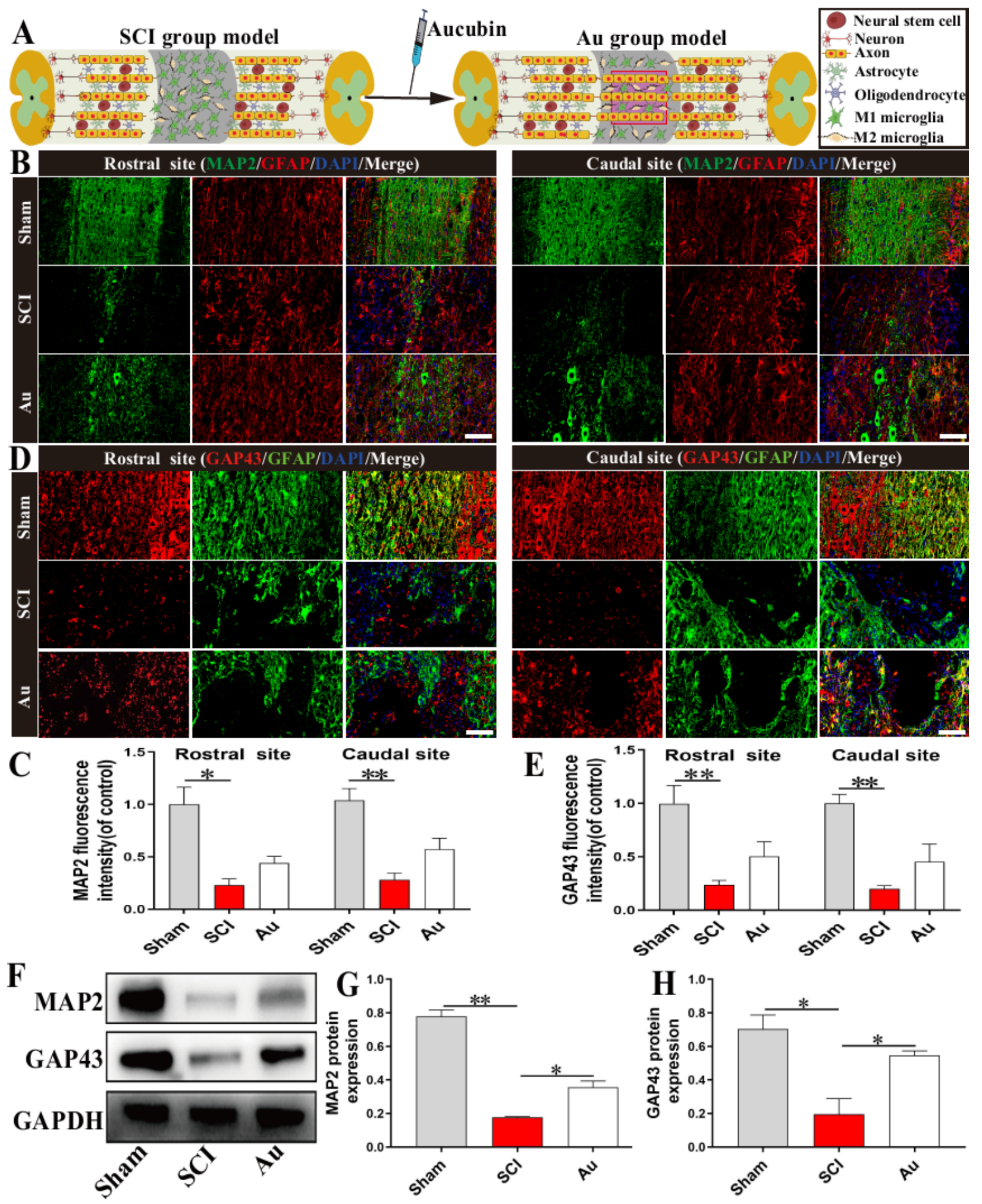

Figure 8

After $\mathrm{SCl}, \mathrm{Au}$ improves neuronal survival and axonal regeneration. (A) Schematic diagram showing that Au improves axon regeneration. (B, C) At 28 days after $\mathrm{SCl}$, double immunofluorescence pictures and 
quantification examination revealed the expression levels of MAP2 (green) and GFAP (red) in each group (scale bar $=100 \mu \mathrm{m})$. (D, E) Representative images containing neurofilament (GAP43) staining and quantitative analysis in each group at 28 days postinjury (scale bar $=100 \mu \mathrm{m}) .(\mathrm{F}, \mathrm{H}) \mathrm{MAP} 2$ and GAP4 proteins were detected and quantified in each group at 28 days postinjury. $\mathrm{N} \geq 3$ per group for separate experiments. ${ }^{*} \mathrm{P}<0.05$ and $* * \mathrm{P}<0.01$.

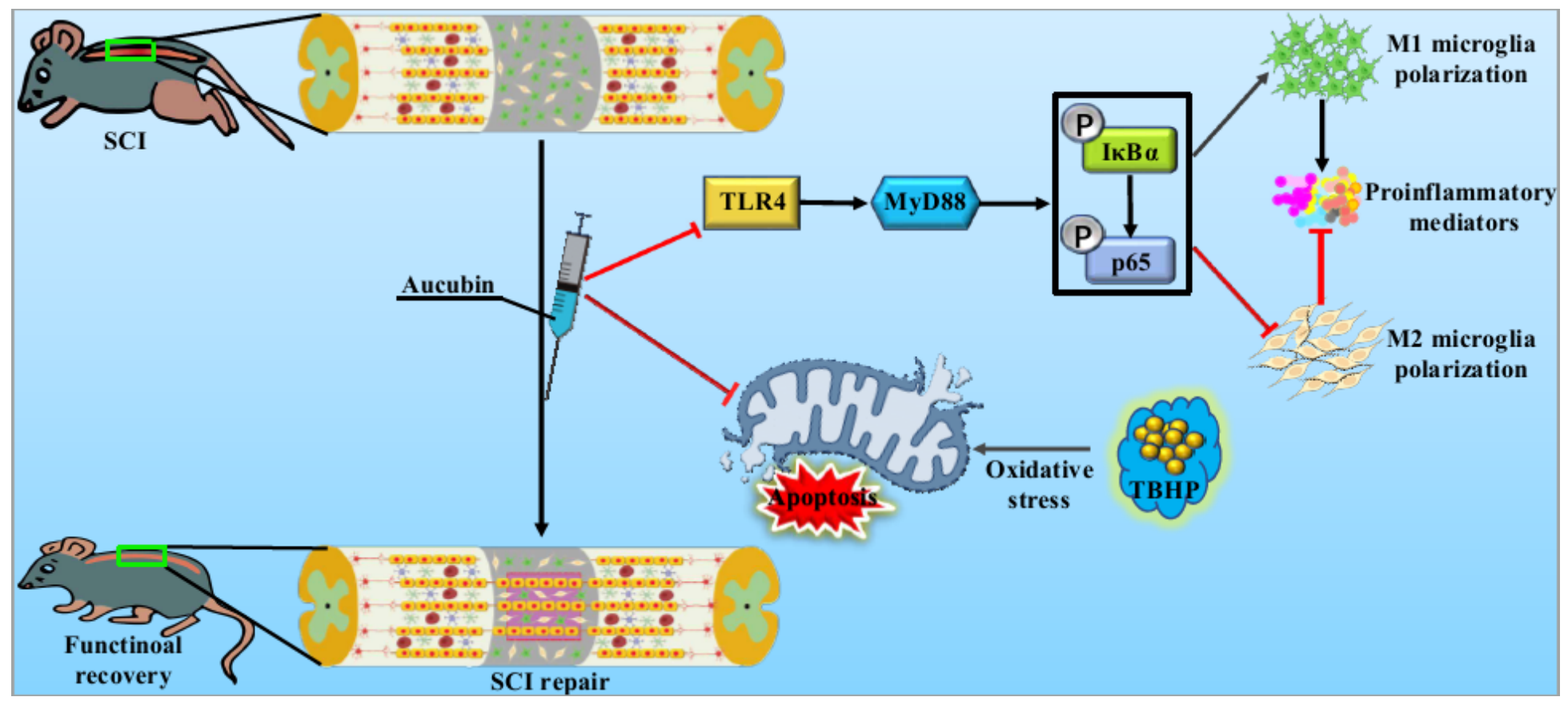

Figure 9

Au's therapeutic effects on $\mathrm{SCl}$ are depicted in this diagram. Au can increase the $\mathrm{M} 2 / \mathrm{M} 1$ cell ratio by inhibiting the TLR4/NF-KB pathway, suppress neuronal apoptosis via inhibit mitochondrial dysfunction, and ultimately promote the functional recovery of rats with $\mathrm{SCl}$. 